\title{
Contextualizing Cognitions: the Relation Between Negative Post-traumatic Cognitions and Post-traumatic Stress Among Palestinian Refugees
}

\author{
H. E. van Heemstra ${ }^{1,2} \cdot$ W. F. Scholte ${ }^{1,3} \cdot$ T. Ehring $^{4}$ • P. A. Boelen ${ }^{1,2}$
}

Published online: 5 February 2020

(C) The Author(s) 2020

\begin{abstract}
Negative post-traumatic cognitions (PTC) are a relevant factor in the development, persistence, and treatment of post-traumatic stress disorder (PTSD). Palestinian refugees live under challenging circumstances and have negative future prospects, so negative cognitions might be expected to prevail. It is uncertain whether findings on the relation between PTC and PTSD in other (non-refugee) populations can be generalized to the Palestinian refugee context. The first objective was to examine the degree to which endorsement of PTC in this sample differed from the endorsement observed in other samples. The second objective was to investigate whether PTC explain variance in PTSD symptomatology and are predictive of PTSD diagnostic status. In Palestinian refugees ( $N=85,51.8 \%$ female), PTSD symptoms and negative cognitions were assessed. One sample $t$ tests and multiple logistic regression analyses were performed. Total PTC scores were significantly higher in the Palestinian sample than in reference samples. Negative cognitions explained significant variance in PTSD symptoms and probable diagnostic status. Findings support the relevance of PTC for PTSD symptoms and diagnosis in a Palestinian refugee sample, in line with the cognitive model for PTSD. This is especially relevant for researchers and clinicians working with refugees in conflict areas.
\end{abstract}

Keywords Cognitions $\cdot$ Trauma $\cdot$ PTSD $\cdot$ Refugees $\cdot$ Conflict area

The number of refugees worldwide is currently increasing (UNHCR 2017). Refugees are challenged by traumatic events and post-migration stressors, which is reflected in high prevalence rates of post-traumatic stress disorder (PTSD) and other mental health problems (Fazel et al. 2005; Giacco et al. 2018; Porter and Haslam 2005; Steel et al.

H. E. van Heemstra

j.van.heemstra@equatorfoundation.nl

Extended author information available on the last page of the article 
2009). Fifty percent of all refugees live in unstable and insecure situations, e.g., refugee camps (Silove et al. 2017), and are at risk for experiencing (additional) trauma (Karunakara et al. 2004).

The current study focuses on a sample of Palestinians residing in refugee camps in a political conflict area. Their living conditions are poor (UNWRA 2015a, b, c, 2017) and unsafe (OCHA 2017). Many are frustrated about current and future prospects (UNWRA 2015b). Their social and political environment causes great physical and psychological pressure (UNWRA 2015a, c), and PTSD prevalence estimates are high (Lavi and Solomon 2005; Madianos et al. 2011). In such circumstances, it is conceivable that negative cognitions about the outside world and its potentially devastating effect on oneself and one's coping resources are common. These negative cognitions may also shape the interpretation of traumatic events as prevailing and unavoidable.

Previous work by Davis et al. 2016 described "negative trauma-related cognitions" three categories: negative cognitions about the self (e.g., I am inadequate), about others (e.g., you can never know who will harm you), and about the world (e.g., the world is a dangerous place). It has been suggested that these cognitions contribute to a sense of 'current threat' and thereby increase the vulnerability for developing PTSD (Ehlers and Clark 2000). Previous studies examining different traumatized populations found strong correlations between these negative trauma-related cognitions and PTSD symptoms (Daie-Gabai et al. 2011; van Emmerik et al. 2006; Müller et al. 2010; Su and Chen 2008). Moreover, negative cognitions have been shown to be predictive for the development (Ehring et al. 2008; Kushner et al. 1993; Foa et al. 1998) and persistence of PTSD (Ehlers et al. 1998) after having experienced a traumatic event. There is also evidence that decrease of PTSD during cognitive behavioral treatment is associated with a reduction of negative cognitions (Brown et al. 2019).

Most studies on the role of negative cognitions for the onset, persistence, and treatment of PTSD were based on Ehlers and Clark's (2000) cognitive model of PTSD. The model posits that PTSD arises as a consequence of - among other factorsnegative interpretations of the traumatic event and/or its consequences, including an overgeneralization of the possibility that the event will occur again, while the danger has actually disappeared and the individual is now objectively safe.

Prior work (e.g., Lancaster et al. 2011) has validated Ehlers and Clark's (2000) model and shown that negative cognitions about oneself tend to have the strongest relation to PTSD symptoms, but that negative cognitions on the world and self-blame are also associated with PTSD symptoms, albeit somewhat lower. It remains to be tested whether the model still holds in populations where people continue to live under harsh and currently threatening conditions (i.e., without objective safety), as it applies to many refugees. They may have negative cognitions resulting from adequate interpretations of the world, based on their daily lives (e.g., The world is a dangerous place) on top of potential overgeneralizations of their traumas. Additionally, their daily challenges can increase vulnerability for negative evaluations regarding themselves (e.g., I am powerless) or regarding self-blame (e.g., I should have behaved differently). This may affect the correlations between negative cognitions and PTSD in at least two ways. On the one hand, the correlations could be weak compared to findings in Western samples, because negative cognitions are commonplace and not exclusive for people with PTSD. On the other hand, negative cognitions elicited by living conditions could increase the vulnerability for PTSD after trauma, because they may impact the 
processing of new traumas, resulting in relatively high levels of PTSD in refugee populations in line with the cognitive model suggested by Ehlers and Clark (2000).

Previous findings indicate that in refugee populations resettled in high-income countries, negative trauma-related cognitions are more prevalent than in local reference groups (Ter Heide et al. 2017) and that these cognitions are associated with PTSD symptoms (Schnyder et al. 2015). Meanwhile, very little is known about the association between negative trauma-related cognitions and PTSD in refugees still living in conflict settings. As cultural differences may impact psychological mechanisms on trauma (e.g., Summerfield 1999), cultural cross-validation of the association between cognitions and PTSD for this population is necessary. One study found that the level of negative cognitions did not predict future development of PTSD symptoms among mothers living in Gaza (Diab et al. 2018). However, this study was conducted in a very specific sample (mothers); therefore, it is uncertain if findings can be generalized to other populations.

The current study aimed to test whether the assumptions of the cognitive model for PTSD regarding a key role of trauma-related appraisals are applicable in a non-Western context, where current threats are a part of daily live. Specifically, we sought to investigate the prevalence of negative trauma-related cognitions and their association with symptoms of PTSD in a sample of Palestinians living in refugee camps. The first aim was to examine the degree to which endorsement of negative trauma-related cognitions about the self, world, and self-blame in this sample differed from the endorsement observed in other (non-refugee) samples. Considering the living circumstances of our sample, we expected significantly higher levels of negative cognitions compared to previously reported samples. The second aim was to examine the association of negative trauma-related cognitions with symptoms of PTSD in our sample, over and above the severity of trauma exposure. In keeping with prior research, it was expected that significant positive associations would emerge. Apart from examining associations with PTSD symptom levels, we also examined the hypothesis that people with probable PTSD (based on their scores on the Harvard Trauma Questionnaire - see below) scored higher on negative cognitions than their counterparts without probable PTSD.

\section{Methods}

\section{Participants}

Participants were all employees or volunteers at the clinics or centers where the study was conducted (see below). Inclusion criteria were (1) aged 18 or older, (2) ability to read and write, and (3) providing informed consent. There were no exclusion criteria.

\section{Procedure}

The current study was conducted in three of the nineteen refugee camps on the West Bank in Palestine. Study locations were a cultural center in Jenin Camp (approximately 16,000 inhabitants) located in Jenin, a clinic in Askar camp (approximately 15,900 inhabitants) in Nablus, and a community center in Balata (approximately 23,600 inhabitants), adjacent to Nablus, or the homes of participants. Data collection took place between April and July 2010. Participants were recruited through the institutes 
where they were working. Depending on the preference of participants, questionnaires were administered in one of the institutes or at their homes.

Prior to questionnaire administration, participants received an information brochure about the research mentioning (1) the study objectives, (2) the fact that participants were free to terminate study participation at any moment, (3) guaranteed anonymization of data, and (4) the absence of compensation for study participation. This information was also read out loud in English in the presence of an independent Arabic interpreter who could translate whenever necessary. Subsequently, participants were invited to sign an informed consent. In order to prevent missing data, the researcher checked the submitted questionnaires directly and asked participants to fill out missing items when applicable. The whole procedure lasted a maximum of $30 \mathrm{~min}$ in total. Responses to open questions were translated by an independent interpreter. The study was approved by the Ethics Committee of the Psychology department of the University of Amsterdam (project number: 2010-KP-1052).

\section{Measures}

Demographic Data Participants provided information on their gender, age, profession, and marital status (i.e., married yes/no).

Post-traumatic Cognitions Inventory The Post-Traumatic Cognitions Inventory (PTCI; Foa et al. 1999) is a self-report questionnaire measuring post-traumatic cognitions. The original English version was translated to Arabic for the current study, and then backtranslated by another independent translator, to ensure equivalence of the two language versions. The PTCI comprises 36 items forming three subscales: negative cognitions about the self (SELF, 21 items, e.g., I'm a weak person), negative cognitions about the world (WORLD, 7 items, e.g., The world is a dangerous place), and self-blame (selfblame, 5 items, e.g., The event happened to me because of the sort of person I am), respectively. The items are scored on a scale from 1 (totally disagree) to 7 (completely agree), resulting in a minimum total score of 36 and a maximum total score of 252 . Prior research has shown that the scale has adequate reliability and convergent and discriminant validity (e.g., Foa et al. 1999). In the current sample, Cronbach's alphas were .96 (total PTCI), .94 (SELF), .85 (WORLD), and .61 (self-blame).

Harvard Trauma Questionnaire The Harvard Trauma Questionnaire (HTQ) is a selfreport questionnaire measuring trauma exposure and the severity of PTSD symptoms. The questionnaire consists of three parts, two of which were used in this study. The HTQ was developed for transcultural use (Mollica et al. 1992), and a translated and cross-culturally adapted Arabic version was used for the current study (Shoeb et al. 2007). Part 1 consists of 17 items listing possible traumatic events (e.g., 'rape', 'murder of family or friends', and 'torture'). There are four response options: 'experienced' (4), 'witnessed' (3), 'heard about it' (2), and 'not applicable' (1). The sum score of all items provides an index for the degree of trauma exposure, with a minimum score of 17 (no events experienced) and a maximum of 68 (all events personally experienced). Part 2 assesses details on the traumatic event, but was not used in this study. Part 3 consists of 30 statements scored on a scale from 1 (not at all) to 4 (extremely). In the current study, we only used the first 16 items, as these are directly derived from the DSM-III-R/DSM- 
IV criteria for PTSD. The other 14 items concern the impact of a traumatic experience on daily life (e.g., 'troubled by bodily pain or physical problems') or cultural idioms of distress ('tired soul'). The decision to delete these items was made because this specific Arabic version was developed for another population than our study sample; therefore, including these specific items outside the DSM-IV category was unsuitable. The items can be subdivided into three subscales corresponding to the DSM-IV-based symptom clusters of PTSD: intrusions (e.g., 'recurring nightmares'), hyperarousal (e.g., 'feeling irritable or having outbursts of anger'), and avoidance (e.g., 'avoiding activities that remind you of the traumatic or hurtful event'). The mean score of part 3 items was used as an index for the severity of the PTSD symptoms.

The HTQ is a suitable screening tool for PTSD, with a cut-off score of 2.5 (e.g., Mollica et al. 1992), based on the first 16 items of part 3, as an established cut-off for the DSM-IV PTSD diagnosis (Wind et al. 2017). The instrument has proven to be valid and reliable (Mollica et al. 2004). In the current sample, Cronbach's alpha of the HTQ part 3 was .88 .

\section{Statistical Analysis}

SPSS Statistics version 23 was used to perform the statistical analyses (IBM, Armonk, NY, USA). The significance threshold was set at $p<.05$ for all tests. One sample $t$ tests were used to compare the PTCI scores for participants in our sample to a (non-refugee) reference sample (Foa et al. 1999). This reference sample $(N=600)$ consisted of American and English treatment-seeking individuals $(n=110)$, individuals recruited from the community $(n=190)$, and undergraduate volunteers $(n=300)$. The total reference sample was divided in four groups, based on their scores on the PostTraumatic Stress Diagnostic Scale (PDS; Foa 1995). Group 1 reported neither traumatic event(s) nor PTSD symptoms above the PDS the cut-off score $(n=162)$, group 2 reported traumatic event(s) with PTSD symptoms under the PDS cut-off score $(n=$ $185)$, group 3 reported traumatic event(s) with PTSD symptoms above PDS the cut-off score $(n=170)$, group $4(n=83)$ reported otherwise and was excluded. Groups 2 and 3 were used as reference groups in our study. Specifically, we compared group 2 to our study participants reporting trauma exposure and scoring under the PTSD cut-off level on the HTQ $(n=59)$ and group 3 with our study participants reporting trauma exposure and scoring above cut-off on the HTQ $(n=26)$. Cohen's $d$ effect sizes were calculated of the differences between the Palestinians and the reference sample.

Pearson correlations were calculated to examine correlations between the different subscales of the PTCI and the HTQ within the Palestinian sample. Subsequently, a hierarchical multiple regression analysis tested four predictors (number of traumatic events experienced and PTCI subscales SELF, WORLD, self-blame), with PTSD symptoms as a dependent variable. Before running the regression analysis, the following assumptions were checked: independence of observations, linear relationship between PTSD symptoms and all predictors independently, homoscedasticity, multi-collinearity, the undermining effect of unusual points, and normal distribution of dependent variable and residuals. To test the predictive value for each of the PTCI subscales on probable diagnoses of PTSD derived from the HTQ, a logistic regression was performed. 


\section{Results}

\section{Participant Characteristics}

Eighty-seven individuals were invited to participate in the study. Two withdrew, indicating that they found several items in the questionnaires too personal. Characteristics of the remaining 85 participants are shown in Table 1.

\section{Comparing PTCI Scores to Reference Samples}

The endorsement of negative cognitions about the self, world, and self-blame in the current study sample was high compared to two reference groups from one sample (see above; Foa et al. 1999). In the Palestinian PTSD group $(n=26), 46.2 \%$ was male, and mean age was $31.9(S D=11.4)$; for the reference sample $(n=185)$, this was $20 \%$ male, mean age $33.1(S D=12.3)$. In the Palestinian non-PTSD group $(n=59), 49.2 \%$ was male, and mean age was $29.7(S D=9.1)$; for the reference sample $(n=170)$, this was

Table 1 Participant characteristics $(N=85)$

\begin{tabular}{lll}
\hline Variable & $N(\%)$ & $M(S D)$ \\
\hline Demographic characteristics & & \\
Female & $44(51.8)$ & $30.38(9.88)$ \\
Age in years & $53(62.4)$ & \\
Married & & \\
Occupational status & $47(55.3)$ & \\
Employed & $16(18.8)$ & \\
Student & $22(25.9)$ & \\
Unemployed & & \\
Clinical characteristics & $26(30.5)$ & $3.23(1.30)$ \\
Above PTSD cut-off score & \\
PTCI scores & & $4.66(1.41)$ \\
SELF & & $3.38(1.31)$ \\
WORLD & & $3.57(1.18)$ \\
Self-blame & & \\
Total & & $2.58(0.57)$ \\
HTQ scores & & $2.32(0.54)$ \\
Exposure & & $2.11(0.57)$ \\
PTSD symptoms & & $2.41(0.70)$ \\
Avoidance & & $2.54(0.62)$ \\
Intrusions & & \\
Hyperarousal & & \\
\hline
\end{tabular}

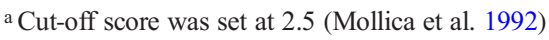

HTQ Harvard Trauma Questionnaire, PTCI Post-Traumatic Cognitions Inventory, SELF subscale negative cognitions on SELF, WORLD negative cognitions on the WORLD 
$42 \%$ male, mean age $25.3(S D=11.7)$. One sample $t$ tests revealed significantly higher scores in our study sample than in the reference groups on all scales except for selfblame in the PTSD group. Huge to small effect sizes were found. See Table 2 for an overview.

\section{Correlations}

As shown in Table 3, the number of traumatic events experienced was significantly positively correlated with PTSD symptom severity, and with negative cognitions about the self. However, no significant correlations with the PTCI subscales WORLD and self-blame were found. Negative cognitions about the self and the world were significantly and substantially correlated with all PTSD subscales. However, self-blame only showed a significant association with hyperarousal but not the other PTSD symptom clusters.

\section{Regression Analysis}

A significant regression equation was found for the model based on four predictors: number of traumatic events experienced (TE), and SELF, WORLD, and self-blame, $F(4,84)=18.05, p<.001$. The model accounted for $47.4 \%$ of variance in symptom levels of PTSD. The PTCI subscales SELF and self-blame (but not WORLD) contributed unique amounts to the explained variance in PTSD levels. Self-blame had a negative relation to PTSD symptoms. Table 4 summarizes the findings of the regression model.

Table 2 Sample $i$ tests

\begin{tabular}{|c|c|c|c|c|c|c|c|}
\hline \multirow[b]{2}{*}{ Variable } & \multicolumn{2}{|c|}{ Palestinian sample } & \multicolumn{5}{|c|}{ Reference sample } \\
\hline & $M(S D)$ & $N$ & $M(S D)$ & $N$ & $t(d f)$ & $p$ & $d$ \\
\hline Non-PTSD group & & 59 & & 185 & & & \\
\hline Total PTCI & $113.73(34.64)$ & & $49.00(23.52)$ & & $14.35(58)$ & $<.001$ & 2.43 \\
\hline SELF & $2.75(0.96)$ & & $1.05(0.63)$ & & $13.58(58)$ & $<.001$ & 2.35 \\
\hline WORLD & $4.26(1.35)$ & & $2.43(1.42)$ & & $10.43(58)$ & $<.001$ & 1.40 \\
\hline Self-blame & $3.24(1.27)$ & & $1.00(1.02)$ & & $13.528(58)$ & $<.001$ & 2.06 \\
\hline PTSD group & & 26 & & 170 & & & \\
\hline Total PTCI & $162.27(39.76)$ & & $133.00(44.17)$ & & $3.75(25)$ & .001 & .67 \\
\hline SELF & $4.29(1.34)$ & & $3.60(1.48)$ & & $2.633(25)$ & .014 & .47 \\
\hline WORLD & 5.57 (1.09) & & $5.00(1.25)$ & & $3.67(25)$ & .013 & .46 \\
\hline Self-blame & $3.69(1.37)$ & & $3.20(1.74)$ & & $1.84(25)$ & .078 & .29 \\
\hline
\end{tabular}

Note: $2.5 ; d=$ Cohen's $d$ non-PTSD group Palestinian sample = participants with Harvard Trauma Questionnaire (HTQ) score <2.5; PTSD group Palestinian sample = participants with HTQ score $\geq 2.5$; non-PTSD group reference sample (Foa et al. 1999) = participants with Psychotrauma Diagnostic Scale (PDS) score $\geq 15$; PTSD group reference sample (Foa et al. 1999) = participants with PDS score $<15$. PTCI $=$ Post-Traumatic Cognitions Inventory; SELF = negative cognitions on SELF; WORLD = negative cognitions on the WORLD 
Table 3 Pearson product-moment correlations

\begin{tabular}{llllllllll}
\hline & 1 & 2 & 3 & 4 & 5 & 6 & 7 & 8 & 9 \\
\hline 1. Number of traumatic events experienced & - & & & & & & & & \\
2. PTSD symptoms & $.42^{*}$ & - & & & & & & & \\
3. Intrusions & $.43^{* *}$ & $.84^{* *}$ & - & & & & & & \\
4. Avoidance & $.30^{* *}$ & $.88^{* *}$ & $.58^{* *}$ & - & & & & & \\
5. Hyperarousal & $.39^{* *}$ & $.88^{* *}$ & $.64^{* *}$ & $.69^{* *}$ & - & & & \\
6. Total score PTCI & $.31^{* *}$ & $.60^{* *}$ & $.54^{* *}$ & $.45^{* *}$ & $.57^{* *}$ & - & & \\
7. SELF & $.34^{*}$ & $.62^{* *}$ & $.57^{* *}$ & $.45^{* *}$ & $.57^{* *}$ & $.97^{* *}$ & - & \\
8. WORLD & .18 & $.49^{* *}$ & $.40^{* *}$ & $.41^{* *}$ & $.48^{* *}$ & $.83^{* *}$ & $.70^{* *}$ & - \\
9. Self-blame & .16 & .19 & .15 & .14 & $.22^{*}$ & $.67^{* *}$ & $.54^{* *}$ & $.51^{* *}$ & - \\
\hline
\end{tabular}

PTCI Post-Traumatic Cognitions Inventory, PTSD Post-Traumatic Stress Disorder, SELF negative cognitions on SELF, WORLD negative cognitions on the WORLD, Self-blame negative cognitions on self-blame

$* p<.05$ (two-tailed) $* * *<.001$ (two-tailed)

\section{Logistic Regression Analysis}

The logistic regression model (with PTCI scales as independent and probable PTSD diagnoses according to the HTQ as dependent variables) was significant, $\chi^{2}(3,85)=$ 32.26, $p<.001$. The model explained $31.6 \%$ of the variance in PTSD classification (Cox \& Snell R Square). Additionally, $78.8 \%$ of cases were classified correctly. The subscale SELF was the only subscale making a unique contribution to the explained variance in PTSD classification (see Table 5).

\section{Discussion}

To our knowledge, this is the first study examining the role of negative cognitions in PTSD among a general sample of Palestinian refugees living under stressful conditions. As hypothesized, we found relatively high levels of negative trauma-related cognitions

Table 4 Multiple regression analysis predicting PTSD symptoms

\begin{tabular}{lccccrr}
\hline Predictor & $B$ & $S E B$ & $\beta$ & $t$ & $p$ & VIF \\
\hline Constant & .97 & .24 & & 4.01 & $<.001$ & \\
TE & .24 & .08 & .25 & 2.86 & .005 & 1.14 \\
SELF & .21 & .05 & .51 & 4.07 & $<.001$ & 2.37 \\
WORLD & .08 & .05 & .21 & 1.76 & .082 & 2.09 \\
Self-blame & -.09 & .04 & -.23 & -2.31 & .023 & 1.48 \\
\hline
\end{tabular}

$B$ unstandardised regression coefficients, $\beta$ standardized regression coefficients, SELF negative cognitions on SELF, WORLD negative cognitions on the WORLD, Self-blame negative cognitions on self-blame, TE number of traumatic events experienced, VIF variance inflation factor 
Table 5 Logistic regression analysis predicting probable diagnoses of PTSD according to the HTQ

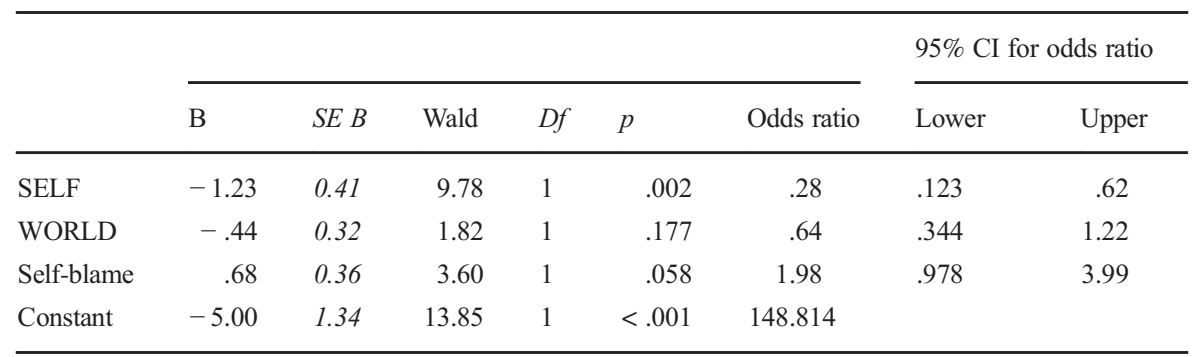

SELF negative cognitions on SELF, WORLD negative cognitions on the WORLD, Self-blame negative cognitions on self-blame

in this sample. Significantly higher PTCI scores were found on the total scale and nearly all subscales compared to Western reference samples (Foa et al. 1999), whereby this applied for Palestinians with and without probable PTSD. Corresponding effect sizes were huge to very large for Palestinians without probable PTSD. For Palestinians with probable PTSD, the effects were nearly medium to medium large, with an exception of a small effect for the self-blame subscale. Additionally, in Palestinians with probable PTSD, negative cognitions on self-blame did not significantly differ from the Western designated reference sample.

Findings indicate that — as hypothesized - the combined three PTCI scales explained significant variance in PTSD symptoms and significantly predicted probable PTSD diagnoses. Notably, the predictive power of negative post-traumatic cognitions was established over and above severity of trauma exposure. At the subscale level, the highest bivariate correlation and a strong unique predictive power was found for negative cognitions about the self. This is in line with earlier findings among Western samples (Karl et al. 2009; Startup et al. 2007).

Although negative cognitions about the world were significantly correlated with PTSD symptoms, they did not significantly predict PTSD symptoms or probable diagnoses in the multiple regression analyses, when taking into account negative cognitions about the self, self-blame, and trauma exposure. Findings from previous studies reveal mixed results concerning the relevance of the world subscale for PTSD (Beck et al. 2004; Müller et al. 2010; Daie-Gabai et al. 2011). In the introduction, we already introduced the idea that negative cognitions about the world may be less strongly related to PTSD in this particular population. Specifically, cognitions Palestinians have about the outside world may be generally negative, due to their daily lives, independent of their levels of PTSD. In other words, in a largely safe and benevolent environment, negative cognitions about the world may be closely and specifically related to PTSD, whereas in an unsafe or uncontrollable environment, these cognitions may be widespread or even normative and therefore related to a much lesser degree to PTSD.

Negative cognitions related to self-blame did not correlate significantly with PTSD symptoms nor with a probable diagnosis for PTSD, contradicting previous findings from refugee populations in Western countries (Schnyder et al. 2015). When controlling for negative cognitions on oneself and the world, self-blame was even associated with lower PTSD symptom severity. This replicates previous findings (e.g., Sheerin et al. 2018; Startup et al. 2007). One intriguing explanation for this could be the 
protective function of a feeling of control originating from behavioral self-blame (Koss et al. 2002), which is part of the subscale measuring self-blame. Such assumption would imply that self-blame could counter-act negative self- and world-views by maintaining a feeling of control. Meanwhile, we should consider that these results may have been compromised by the low Crohnbach's alpha for the self-blame subscale, as outlined in previous work (e.g., Beck et al. 2004; Müller et al. 2010). As the current study is not the first to find a negative relation between self-blame and PTSD symptoms (e.g., Sheerin et al. 2018; Startup et al. 2007), it is important to study the role of selfblame for PTSD in more detail and make sure that instruments are adequate.

In conclusion, the current study supports the view that negative trauma-related cognitions are associated with PTSD symptoms, which confirms previous findings from Western samples (Beck et al. 2004; van Emmerik et al. 2006; Foa et al. 1999). However, this association was mainly found for negative cognitions about the self, not for negative cognitions about the world. For self-blame, a more complex pattern emerged with a significant negative association to PTSD symptoms.

This finding suggests that even in the context of objective instability and lack of safety, negative interpretations of the traumatic event and/or its consequences, concerning oneself and partly concerning self-blame, are associated to PTSD symptoms and diagnosis, in line with the cognitive model of PTSD (Ehlers and Clark 2000). Additionally, negative cognitions about oneself appear to be of particular importance as they show more consistent associations than negative cognitions on the world and on self-blame; this also confirms earlier reports (e.g., Kolts et al. 2004; Moser et al. 2007).

The current study has several limitations. First, the study entails a cross-sectional design which makes it impossible to draw conclusions regarding the causality between predictor and dependent variable. Second, although the HTQ cut-off score of 2.5 for clinically significant PTSD is being used worldwide and in comparable samples (Farhood and Dimassi 2012), its transcultural metric and scale invariance have been found to be questionable.

Reassuringly, however, this is mainly relevant when comparing PTSD levels between different cultural groups (Rasmussen et al. 2015), which was not part of our study. Thirdly, the validity of the self-blame subscale has been under discussion due to its measurement invariances and weak factor loadings in previous studies (Müller et al. 2010; Startup et al. 2007). These first limitations call for modesty towards our findings. Fourth, the PTCI subscales vary in the number of items which may result in a suppressor effect of the more extended subscale SELF on the other scales with limited items (Startup et al. 2007). Elevations about the self might overlap with cognitions about self-blame and the world and could therefore possibly suppress existing associations between self-blame and PTSD. Due to the relatively high number of items in the SELF subscale, this overlap would primarily affect the predictive value of the other scales with less items. Fifth, the prevalence of current psychosocial stressors is generally high in Palestinian refugee camps but was not controlled for as a predictor variable in this study. Although a high level of post-traumatic cognitions was found in our sample, it is impossible to determine if these appraisals are indeed related to external circumstances. In future studies, it would be useful to directly examine the interaction between social stressors and cognitions, and how this interaction relates to PTSD. Sixth, because we assessed PTSD as defined in DSM-IV, our findings may not necessarily generalize to PTSD as currently defined in DSM-5. Seventh, both questionnaires have not been validated for the study sample. However, the PTCI (Diab et al. 2018) and HTQ (e.g., Punamäki et al. 2002) have been used before in Palestinian samples. An eighth limitation is the relatively small 
sample size. Although it meets rule-of-thumb criteria (e.g., Harris 1985), it cannot be ruled out that type II error (Faber and Fonseca 2014) may be responsible for nonsignificant findings (e.g., on the predictive value of negative cognitions about the world) due to limited power. Future research should therefore replicate in larger samples. Lastly, because we had no access to the dataset of the western reference sample, we were unable to include these in a formal moderation analysis.

Notwithstanding these limitations, our study extends earlier research by showing that the predictive value of negative cognitions on PTSD symptoms and classifications stands firmly within a population living under conflict and presenting high levels of negative traumarelated cognitions. Based on our findings, we tentatively recommend addressing negative cognitions, especially about the self, in working with populations living under conflict and suffering from PTSD. Future studies should test the effectiveness of cognitive therapy for PTSD among populations living under conflict. Also, we recommend including a higher number of participants in future studies, in order to enable analysis on the interactions between the different categories of negative cognitions. Moreover, it appears preferable for future research to examine trauma exposure not only in terms of the number of different types of trauma participants experienced, but also in terms of the number of times they were (repeatedly) exposed to trauma, in order to gain a clear picture of the role of this variable. Additionally, it is important to further investigate cultural and social influences on the content of trauma-related cognitions in cultures other than in Western samples. This could help to identify relevant mechanisms that accompany or underlie negative cognitions, and should be included in local cognitive therapies. This is especially relevant for researchers and clinicians working with refugees in conflict areas.

Acknowledgments We thank all participants for their willingness to participate in the study. We thank Ahmad alNimer, for his support during the data collection. We thank Nexhmedin Morina for his support in designing the study. We thank Ilona van Erck for the prepatory work. We thank Niels van der Aa for his (methodological) advice.

Funding Information This study is financially supported by Arq Psychotrauma Expertise Centre.

\section{Compliance with Ethical Standards}

Conflict of Interests The authors declare that they have no conflicts of interest.

Open Access This article is licensed under a Creative Commons Attribution 4.0 International License, which permits use, sharing, adaptation, distribution and reproduction in any medium or format, as long as you give appropriate credit to the original author(s) and the source, provide a link to the Creative Commons licence, and indicate if changes were made. The images or other third party material in this article are included in the article's Creative Commons licence, unless indicated otherwise in a credit line to the material. If material is not included in the article's Creative Commons licence and your intended use is not permitted by statutory regulation or exceeds the permitted use, you will need to obtain permission directly from the copyright holder. To view a copy of this licence, visit http://creativecommons.org/licenses/by/4.0/.

\section{References}

Beck, J. G., Goffey, S. F., Palyo, S. A., Gudmundsdottir, B., Miller, L. M., \& Colder, C. R. (2004). Psychometric properties of the posttraumatic cognitions inventory (PTCI). A replication with motor 
vehicle accident survivors. Psychological Assessment, 16(3), 289-298. https://doi.org/10.1037/10403590.16.3.289.

Brown, L. A., Belli, G. M., Asnaani, A., \& Foa, E. B. (2019). A review of the role of negative cognitions about oneself, others, and the world in the treatment of PTSD. Cognitive Therapy and Research, 43(1), 143-173. https://doi.org/10.1007/s10608-018-9938-1.

Daie-Gabai, A., Aderka, I. M., Allon-Schindel, I., Foa, E. B., \& Gilboa-Schechtman, E. (2011). Posttraumatic cognitions inventory (PTCI): Psychometric properties and gender differences in an Israeli sample. Journal of Anxiety Disorders, 25(2), 266-271. https://doi.org/10.1016/j.janxdis.2010.09.012.

Davis, L. W., Leonhardt, B. L., Siegel, A., Brustuen, B., Luedtke, B., Vohs, J. L., et al. (2016). Metacognitive capacity predicts severity of trauma-related dysfunctional cognitions in adults with posttraumatic stress disorder. Psychiatry Research, 237, 182-187. https://doi.org/10.1016/j.psychres.2016.01.045.

Diab, S. Y., Isosävi, S., Qouta, S. R., Kuittinen, S., \& Punamäki, R. L. (2018). The protective role of maternal posttraumatic growth and cognitive trauma processing among Palestinian mothers and infants. Infant Behavior \& Development, 50, 284-299. https://doi.org/10.1016/j.infbeh.2017.05.008.

Ehlers, A., \& Clark, D. M. (2000). A cognitive model of posttraumatic stress disorder. Behaviour Research and Therapy, 38(4), 319-345. https://doi.org/10.1016/S0005-7967(99)00123-0.

Ehlers, A., Mayou, R. A., \& Bryant, B. (1998). Psychological predictors of chronic posttraumatic stress disorder after motor vehicle accidents. Journal of Abnormal Psychology, 107(3), 508-519. https://doi. org/10.1037/0021-843X.107.3.508.

Ehring, T., Ehlers, A., \& Glucksman, E. (2008). Do cognitive models help in predicting the severity of posttraumatic stress disorder, phobia, and depression after motor vehicle accidents? A prospective longitudinal study. Journal of Consulting and Clinical Psychology, 76(2), 219-230. https://doi. org/10.1037/0022-006X.76.2.219.

Faber, J., \& Fonseca, L. M. (2014). How sample size influences research outcomes. Dental Press Journal of Orthodontics, 19(4), 27-29. https://doi.org/10.1093/ejo/cjp107.

Farhood, L. F., \& Dimassi, H. (2012). Prevalence and predictors for post-traumatic stress disorder, depression and general health in a population from six villages in South Lebanon. Social Psychiatry and Psychiatric Epidemiology, 47(4), 639-649. https://doi.org/10.1007/s00127-011-0368-6.

Fazel, M., Wheeler, J., \& Danesh, J. (2005). Prevalence of serious mental disorder in 7000 refugees resettled in western countries: a systematic review. Lancet, 365(9467), 1309-1314. https://doi.org/10.1016/S01406736(05)61027-6.

Foa, E. B. (1995). Posttraumatic Stress Diagnostic Scale. National Computer Systems.

Foa, E. B., Steketee, G., \& Rothbaum, B. O. (1989). Behavioral-cognitive conceptualizations of post-traumatic stress disorder. Behavior Therapy, 20(2), 155-176. https://doi.org/10.1016/S0005-7894(89)80067-X.

Foa, E. B., Ehlers, A., Clark, D. M., Tolin, D. F., \& Orsillo, S. M. (1999). The posttraumatic cognitions inventory (PTCI): development and validation. Psychological Assessment, 11(3), 303-314. https://doi. org/10.1037/1040-3590.11.3.303.

Giacco, D., Laxhman, N., \& Priebe, S. (2018). Prevalence of and risk factors for mental disorders in refugees. Seminars in Cell \& Developmental Biology, 77, 144-152. https://doi.org/10.1016/j.semcdb.2017.11.030.

Harris, R. J. (1985). A primer of multivariate statistics (2nd ed.). New York: Academic Press.

Karl, A., Rabe, S., Zöllner, T., Maercker, A., \& Stopa, L. (2009). Negative self-appraisals in treatment-seeking survivors of motor vehicle accidents. Journal of Anxiety Disorders, 23(6), 775-781. https://doi. org/10.1016/j.janxdis.2009.03.001.

Karunakara, U. K., Neuner, F., Schauer, M., Singh, K., Hill, K., Elbert, T., \& Burnham, G. (2004). Traumatic events and symptoms of post-traumatic stress disorder amongst Sudanese nationals, refugees and Ugandans in the West Nile. African Health Siences, 4(2), 83-93.

Kolts, R. L., Robinson, A. M., \& Tracy, J. J. (2004). The relationship of sociotropy and autonomy to posttraumatic cognitions and PTSD symptomatology in trauma survivors. Journal of Clinical Psychology, 60(1), 53-63. https://doi.org/10.1002/jclp.10193.

Koss, M. P., Figueredo, A. J., \& Prince, R. J. (2002). Cognitive mediation of rape's mental, physical and social health impact: Tests of four models in cross-sectional data. Journal of Consulting and Clinical Psychology, 70(4), 926-941. https://doi.org/10.1037/0022006X.70.4.926.

Kushner, M. G., Riggs, D. S., Foa, E. B., \& Miller, S. M. (1993). Perceived controllability and the development of posttraumatic stress disorder (PTSD) in crime victims. Behaviour Research and Therapy, 31(1), 105-110. https://doi.org/10.1016/0005-7967(93)90048-Y.

Lancaster, S. L., Rodriguez, B. F., \& Weston, R. (2011). Path analytic examination of a cognitive model of PTSD. Behaviour Research and Therapy, 49(3), 194-201. https://doi.org/10.1016/j.brat.2011.01.002. 
Lavi, T., \& Solomon, Z. (2005). Israeli youth in the second intifada: PTSD and future orientation. Journal of the American Academy of Child and Adolescent Psychiatry, 44(11), 1167-1175. https://doi.org/10.1097 /01.chi.0000177325.47629.4c.

Madianos, M. G., Sarhan, A. L., \& Koukia, E. (2011). Major depression across West Bank: A cross-sectional general population study. The International Journal of Social Psychiatry, 58(3), 315-322.

Mollica, R. F., Caspi-Yavin, Y., Bollini, P., Truong, T., Tor, S., \& Lavelle, J. (1992). The Harvard trauma questionnaire: Validating a cross-cultural instrument for measuring torture, trauma, and posttraumatic stress disorder in Indochinese refugees. Journal of Nervous and Mental Disease, 180(2), 111-116. https://doi.org/10.1097/00005053-199202000-00008.

Mollica, R. F., McDonald, L. S., Massagli, M. P., \& Silove, D. (2004). Measuring trauma, measuring torture: Instructions and guidance on the utilization of the Harvard program in refugee Trauma's versions of the Hopkins symptom Checklist-25 (HSCL-25) \& the Harvard trauma questionnaire (HTQ). Harvard Program in Refugee Trauma.

Moser, J. S., Hajcak, G., Simons, R. F., \& Foa, E. B. (2007). Posttraumatic stress disorder symptoms in trauma-exposed college students: The role of trauma-related cognitions, gender, and negative affect. Journal of Anxiety Disorders, 21(8), 1039-1049. https://doi.org/10.1016/j.janxdis.2006.10.009.

Müller, J., Wessa, M., Rabe, S., Dörfel, D., Knaevelsrud, C., Flor, H., et al. (2010). Psychometric properties of the posttraumatic cognitions inventory (PTCI) in a German sample of individuals with a history of trauma. Psychological Trauma Theory Research Practice and Policy, 2(2), 116-125. https://doi. org/10.1037/a0018603.

OCHA. (2017). Global Humanitarian Overview 2017. Retrieved from https://www.unocha. org/publication/global-humanitarian-overview/global-humanitarian-overview-2017.

Porter, M., \& Haslam, N. (2005). Predisplacement and postdisplacement factors associated with mental health of refugees and internally displaced persons: a meta-analysis. JAMA, 294(5), 602-612. https://doi. org/10.1001/jama.294.5.602.

Punamäki, R. L., Kanninen, K., Qouta, S., \& El-Sarraj, E. (2002). The role of psychological defences in moderating between trauma and post-traumatic symptoms among Palestinian men. International Journal of Psychology, 37(5), 286-296. https://doi.org/10.1080/00207590244000133.

Rasmussen, A., Verkuilen, J., Ho, E., \& Fan, Y. (2015). Posttraumatic stress disorder among refugees: measurement invariance of Harvard trauma questionnaire scores across global regions and response patterns. Psychological Assessment, 27(4), 1160-1170. https://doi.org/10.1037/pas0000115.

Schnyder, U., Müller, J., Morina, N., Schick, M., Bryant, R. A., \& Nickerson, A. (2015). A comparison of DSM-5 and DSM-IV diagnostic criteria for posttraumatic stress disorder in traumatized refugees. Journal of Traumatic Stress, 28(4), 267-274. https://doi.org/10.1002/jts.22023.

Sheerin, C. M., Chowdhury, N., Lind, M. J., Kurtz, E. D., Rappaport, L. M., Berenz, E. C., Brown, R. C., Pickett, T., McDonnald, S. D., Danielson, C. K., \& Amstadter, A. B. (2018). Relation between coping and posttrauma cognitions on PTSD in a combat-trauma population. Military Psychology, 30(2), 98-107. https://doi.org/10.1080/08995605.2017.1420980.

Shoeb, M., Weinstein, H., \& Mollica, R. (2007). The Harvard trauma questionnaire: adapting a cross-cultural instrument for measuring torture, trauma and posttraumatic stress disorder in Iraqi refugees. The International Journal of Social Psychiatry, 53(5), 447-463. https://doi.org/10.1177/0020764007078362.

Silove, D., Ventevogel, P., \& Rees, S. (2017). The contemporary refugee crisis: an overview of mental health challenges. World Psychiatry, 16(2), 130-139. https://doi.org/10.1002/wps.20438.

Startup, M., Makgekgenene, L., \& Webster, R. (2007). The role of self-blame for trauma as assessed by the posttraumatic cognitions inventory (PTCI): a self-protective cognition? Behaviour Research and Therapy, 45(2), 395-403. https://doi.org/10.1016/j.brat.2006.02.003.

Steel, Z., Chey, T., Silove, D., Marnane, C., Bryant, R. A., \& Van Ommeren, M. (2009). Association of torture and other potentially traumatic events with mental health outcomes among populations exposed to mass conflict and displacement: a systematic review and meta-analysis. Jama, 302(5), 537-549. https://doi. org/10.1001/jama.2009.1132.

Su, Y.-J., \& Chen, S.-H. (2008). A three-month prospective investigation of negative cognitions in predicting posttraumatic stress symptoms: the mediating role of traumatic memory quality. Chinese Journal of Psychology, 50(2), 167-186.

Summerfield, D. (1999). A critique of seven assumptions behind psychological trauma programmes in waraffected areas. Social Science \& Medicine, 48(10), 1449-1462. https://doi.org/10.1016/S0277-9536(98 )00450-X.

Ter Heide, F. J. J., Sleijpen, M., \& van der Aa, N. (2017). Posttraumatic world assumptions among treatmentseeking refugees. Transcultural Psychiatry, 54(5-6), 824-839. https://doi.org/10.1177 /1363461517741811. 
United Nations High Commissioner for Refugees. (2017). Global trends: Forced displacement in 2017. Retrieved from: http://www.unhcr.org/5b27be547.pdf\#zoom=95

United Nations Office for the Coordination of Humanitarian Affairs. (2017). Occupied Palestinian territory: Monthly figures. Retrieved from: https://www.ochaopt.org/content/monthly-figures.

United Nations Relief and Works Agency for Palestine Refugees. (2015a). Askar camp. Retrieved from: https://www.unrwa.org/sites/default/files/askar_refugee_camp.pdf.

United Nations Relief and Works Agency for Palestine Refugees. (2015b). Balata camp. Retrieved from: https://www.unrwa.org/sites/default/files/balata refugee camp.pdf.

United Nations Relief and Works Agency for Palestine Refugees. (2015c). Jenin camp. Retrieved from: https://www.unrwa.org/sites/default/files/jenin_refugee_camp.pdf.

van Emmerik, A., Schoorl, M., Emmelkamp, P., \& Kamphuis, J. H. (2006). Psychometric evaluation of the Dutch version of the posttraumatic cognitions inventory (PTCI). Behaviour Research and Therapy, 44(7), 1054-1065. https://doi.org/10.1016/j.brat.2005.07.002.

Wind, T. R., van der Aa, N., de la Rie, S., \& Knipscheer, J. (2017). The assessment of psychopathology among traumatized refugees: measurement invariance of the Harvard trauma questionnaire and the Hopkins symptom Checklist-25 across five linguistic groups. European Journal of Psychotraumatology, 8(S2), 1321357. https://doi.org/10.1080/20008198.2017.1321357.

Publisher's Note Springer Nature remains neutral with regard to jurisdictional claims in published maps and institutional affiliations.

\section{Affiliations}

\section{H. E. van Heemstra ${ }^{1,2} \cdot$ W. F. Scholte ${ }^{1,3} \cdot$ T. Ehring $^{4}$ • P. A. Boelen ${ }^{1,2}$}

1 ARQ Centrum'45, ARQ National Psychotrauma Centre, Diemen, Netherlands

2 Department of Clinical Psychology, Utrecht University, Utrecht, Netherlands

3 Department of Psychiatry, Amsterdam UMC, University of Amsterdam, Amsterdam, Netherlands

4 Department of Psychology, LMU Munich, Munich, Germany 